\title{
Theory-based learning analytics to explore student engagement patterns in a peer review activity
}

\author{
Erkan Er \\ Universidad de Valladolid \\ Dragan Gasevic \\ Monash University
}

\author{
Cristina Villa-Torrano \\ Universidad de Valladolid \\ Miguel L. Bote-Lorenzo
Universidad de Valladolid
}

\author{
Yannis Dimitriadis \\ Universidad de Valladolid \\ Juan I. Asensio-Pérez \\ Universidad de Valladolid
}

\author{
Eduardo Gómez-Sánchez \\ Universidad de Valladolid
}

\begin{abstract}
Peer reviews offer many learning benefits. Understanding students' engagement in them can help design effective practices. Although learning analytics can be effective in generating such insights, its application in peer reviews is scarce. Theory can provide the necessary foundations to inform the design of learning analytics research and the interpretation of its results. In this paper, we followed a theory-based learning analytics approach to identifying students' engagement patterns in a peer review activity facilitated via a webbased tool called Synergy. Process mining was applied on temporal learning data, traced by Synergy. The theory about peer review helped determine relevant data points and guided the top-down approach employed for their analysis: moving from the global phases to regulation of learning, and then to micro-level actions. The results suggest that theory and learning analytics should mutually relate with each other. Mainly, theory played a critical role in identifying a priori engagement patterns, which provided an informed perspective when interpreting the results. In return, the results of the learning analytics offered critical insights about student behavior that was not expected by the theory (i.e., low levels of co-regulation). The findings provided important implications for refining the grounding theory and its operationalization in Synergy.
\end{abstract}

\section{CCS CONCEPTS}

- Applied computing $\rightarrow$ Education; Interactive learning environments; • Information systems $\rightarrow$ Information systems applications; Data mining.

\section{KEYWORDS}

Peer reviews, learning analytics, student engagement, process mining

\section{ACM Reference Format:}

Erkan Er, Cristina Villa-Torrano, Yannis Dimitriadis, Dragan Gasevic, Miguel L. Bote-Lorenzo, Juan I. Asensio-Pérez, Eduardo Gómez-Sánchez,

Permission to make digital or hard copies of all or part of this work for personal or classroom use is granted without fee provided that copies are not made or distributed for profit or commercial advantage and that copies bear this notice and the full citation on the first page. Copyrights for components of this work owned by others than ACM must be honored. Abstracting with credit is permitted. To copy otherwise, or republish, to post on servers or to redistribute to lists, requires prior specific permission and/or a fee. Request permissions from permissions@acm.org.

LAK21, April 12-16, 2021, Irvine, CA, USA

(C) 2021 Association for Computing Machinery.

ACM ISBN 978-1-4503-8935-8/21/04 . \$15.00

https://doi.org/10.1145/3448139.3448158

\author{
Alejandra Martínez Monés \\ Universidad de Valladolid
}

and Alejandra Martínez Monés. 2021. Theory-based learning analytics to explore student engagement patterns in a peer review activity. In LAK21: 11th International Learning Analytics and Knowledge Conference (LAK21), April 12-16, 2021, Irvine, CA, USA. ACM, New York, NY, USA, 11 pages. https://doi.org/10.1145/3448139.3448158

\section{INTRODUCTION}

Peer review is an effective strategy that can contribute to the learning process of students [10]. Students can learn from different perspectives brought by their peers, improve their communication skills, and reflect on their work by reading others' ideas. However, students' engagement in the activity is a key element that determines the extent to which the intended learning gains are achieved [16]. For example, students can achieve higher learning gains if they attend the peer feedback received (e.g., asking questions to make meaning from it) [1] and make a concrete plan about the revisions for improving their work [3]. There might be numerous learning processes with which students engage while being reviewed. Varying levels of behavioral engagement in these processes and specific strategies followed can help determine to what degree student behavior matches what is pedagogically intended. Therefore, a comprehensive understanding of student engagement patterns can help derive implications for improving peer review practices.

Despite the importance of student engagement, existing research has mostly focused on the relationship between various students' perceptions regarding peer reviews (e.g., perceived effectiveness of peer assessment, perceptions of the peer review process) [8, 9]. On the other hand, with the increasing digitalization in higher education, peer reviews often take place online, which creates richer affordances for tracing fine-grained temporal learning data that can be used to detect actual peer-review engagement. The field of learning analytics offers tools and techniques to process such learning data to derive useful insights about student behavior. Regardless of this immense opportunity, there is a scarce amount of research on the use of learning analytics to study and understand how students engage in peer reviews.

In spite of the advances in the field of learning analytics in helping understand and support learning processes, there are increasing concerns regarding the rigorousness of theory-free learning analytics approaches $[11,17]$. Grounding learning analytics in theories can offer two main benefits among many others. First, the larger the learning data collected, the more insights can be derived about student behavior, but only if it is known where to look at among 
hundreds of data points. Theory can provide a foundation for researchers to make informed decisions on including or excluding the data points or variables in their analysis of a learning behavior [17]. Similarly, the use of theory can help determine beforehand what learner actions to trace. Second, theory can provide a frame for interpreting the results of learning analytics based on a priori patterns that are expected and critical to happen according to the theory and for identifying unexpected patterns that are not predicted by the theory [11]. In this way, learning analytics would afford not only to validate the theory but also to further refine it based on how student behavior deviates from what is theorized.

This study aimed to apply theory-based learning analytics to explore how student engagement unfolds over time in a peer review activity facilitated via a web-based tool called Synergy. Within the scope of this paper, we report the results regarding the behavior of reviewed students. The theoretical framework (TF) grounding this study was proposed in [3] to conceptualize collaborative peer feedback and was used to inform the design of the peer review activity as implemented in Synergy. The learning data traced by Synergy based on the actions taken by the students were analyzed. We applied process mining on the data, an often used technique to extract patterns from temporal learning data [5], to identify the (reviewed) students' engagement patterns based on their performance levels. Previous research suggests differences in students' engagement based on their performance levels. For example, in [2], the researchers found that high performing learners have more similar temporal and sequential ordering of regulation processes as theorized than the ordering of low performers. Similarly, we also expect that high performers' engagement patterns would be closely aligned with the TF.

According to the TF, all peer review processes involved in Synergy are associated with a certain phase and type of regulation of learning, and certain temporal and sequential relations exist between the relevant processes of peer review. These theorized relations were compared with the engagement patterns identified by process mining to identify the degree to which the observed patterns may coincide with or differ from the ones that were induced by the TF. Accordingly, this study aims to answer the following research question:

- How can theory-informed LA help identify and interpret engagement patterns in peer reviews?

\section{BACKGROUND}

This section provides background information about the grounding theory and its operationalization in Synergy.

\subsection{A theoretical framework of collaborative peer feedback}

Synergy is founded in a theoretical framework (TF) of collaborative peer feedback proposed in [3]. This framework conceptualizes peer feedback as a collaborative activity involving peers' systematic interactions organized by three dialogic phases. In the first phase, reviewing peers work together to plan and coordinate the feedback provision. During this phase, the involvement of the reviewed students in the assessment and discussion of the quality of the work is recommended. Their participation in this phase is aimed to support reaching a consensus on the weaknesses and strengths of the work. Second phase mainly involves feedback provision and discussion, in which, reviewed students identify relevant actions to take based on the peer feedback received. In the third phase, the reviewed students take the planned actions to improve their learning and to progress on their work reviewed.

These phases require different types of learning regulation. While in the first phase students socially regulate their learning (SSRL) to align their perspectives about the quality of the work and determine the focus of feedback, in the second phase students self-regulate their learning (SRL) by identifying relevant learning actions based on the feedback received and co-regulate (Co-RL) by discussing with the reviewing peers to make sense of the feedback and to ensure that the actions identified match the feedback received. Similar to the second phase, the last phase also involves SRL and Co-RL as students track and monitor their progress on the actions and discuss with the peers any issues faced while performing the actions. Several principles are proposed in [3] to help practitioners operationalize this TF in practice. These principles, mapped with the phases of peer feedback, are presented in Table 1

\subsection{Synergy}

Synergy is a web-based platform strongly rooted in the TF presented in the section above. Aligned with the phases in the grounding framework, Synergy facilitates peer reviews in three main stages. In each of these stages, the principles presented in Table 1 are used to inform the design of Synergy. The page showing the main review task for reviewed students is displayed in Figure 1. In this first phase, reviewed students perform a self-assessment using a rubric (created by the instructor within Synergy) (see Figure 2a). This interface is designed based on the principle \#1. Once the assessment is completed, students can compare their self-assessment scores with the assessment scores assigned by all peers as seen in Figure 2b. They can click the "Discuss" button to post any comments about the scores (e.g., sharing their perspective to reach a consensus). This discussion feature is added to Synergy considering the principle \#2.

In the second stage, reviewing peers post and discuss feedback with the students. Feedback provision and discussion are facilitated through Google Docs. During feedback discussion, students can create learning actions in Synergy (see Figure 3) to plan the revisions on their work. To do so, Synergy provides a specific form, where students need to enter a short description of the action, indicate the corresponding feedback, and determine a completion date and difficulty level. The actions created are displayed on the same page. This functionality is added based on the principle \#5. In the last phase, students are responsible for revising their work based on the action plan they created previously. Synergy uses Google Docs to allow the students to incorporate the revisions guided by the actions (see Figure 4a). The list of actions created previously are listed on the same page, where students can update the ongoing progress for the actions according to the advancements in revising their work (see Figure 4b), as suggested by the principle \#6.

Moreover, as shown in Figure 5, Synergy allows students to check the history of progress updates for a selected action (indicated with blue line) along with the revision history (indicated with grey line), which is accessible through clicking on the "Monitor" button 
Table 1: Principles for designing effective collaborative peer feedback practices

\begin{tabular}{|c|c|}
\hline Phases of peer feedback & Principles \\
\hline $\begin{array}{l}\text { Phase 1: Aligning the perspectives and } \\
\text { planning the feedback (SSRL) }\end{array}$ & $\begin{array}{l}\text { Connect self-evaluation with peer evaluations. } \\
\text { Provide opportunities to resolve the discrepancies in students' perspectives about the quality } \\
\text { of the work. } \\
\text { Provide mechanisms to (collectively) plan the feedback before its provision }\end{array}$ \\
\hline $\begin{array}{l}\text { Phase 2: Discussion of the feedback to } \\
\text { support its uptake (Co-RL and SRL) } \\
\text { Phase 3: Translation of the feedback } \\
\text { into task engagement and progress } \\
\text { (Co-RL and SRL) }\end{array}$ & $\begin{array}{l}\text { Enable dialogue around the feedback to support its uptake. } \\
\text { Enable students to set goals and create an action plan with peers based on the feedback. } \\
\text { Enable students (both reviewing and the reviewed students) to track, monitor, and evaluate } \\
\text { their progress on the learning actions. } \\
\text { Enable dialogue with peers while students are revising their work. }\end{array}$ \\
\hline
\end{tabular}

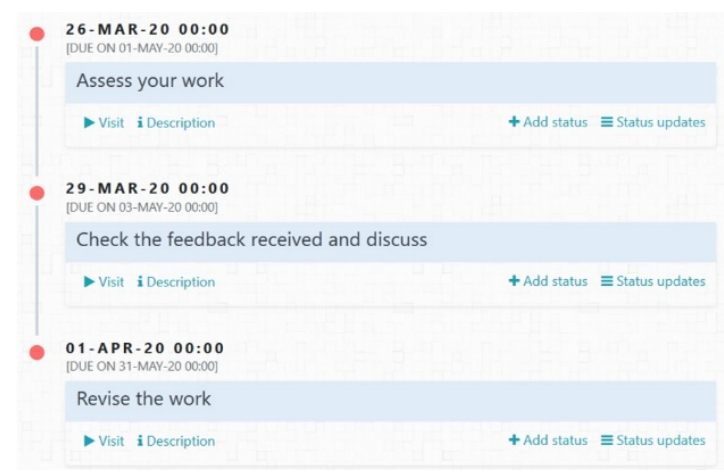

Figure 1: The page showing the review tasks for students being reviewed

corresponding to the action. This feature was grounded in the principle \#6 to enhance students' capacities to monitor the progress. Following the principle \#7, Synergy contains a discussion feature for ongoing actions to promote dialogue among students about the correct and effective use of the peer feedback.

\section{THE STUDY}

\subsection{Context and participants}

The study took place at a postgraduate course on technology enhanced distance learning during the spring semester of 2020 in a European university. Thirty preservice teachers attended the course from various disciplines including Computer Science, Preschool and Elementary Education, Physics, and Mathematics. The students had limited prior knowledge on learning design (which was the focus of the course assignment) and limited experience in peer reviews. Students worked in groups (of 3 or 4 ) to carry out a learning design project as the main course assignment. The submitted learning designs were delivered as Google Documents and later peer reviewed using Synergy. In all phases of the peer review, discussions were kept optional. The final revisions on the group work were performed outside Synergy and no data were available about the engagement in the revisions. The peer review activity lasted 3 weeks. Two or three peers were randomly assigned to review each group submission.
Students were (posteriorly) categorized into two groups based on their performance levels. The grades of the reviewed projects (ranging from 0 to 5 ) were used as the performance variable. These grades were assigned by the course instructors based on the quality of the final learning designs. The division was performed based on the 25th percentile of their grades which resulted in two groups: high performers $(n=18)$, who had a score greater than 4 points (mean: 4.70, and standard deviation: 0.24 ), and the medium performers $(n=12)$, who had scores that were equal to or less than 4 points (mean: 3.70, and standard deviation: 1.12). As the sample size was small and there were almost no failed students, the term "low performers" was avoided.

\subsection{Data and coding}

In Synergy, each time a student performs a click, the corresponding action is automatically recorded on an online database. It is critical to clarify that the actions recorded comprise all the activities performed by the students, which include creating learning actions based on peer feedback in Synergy (see section 2.2). These clickstream data generated during the peer review activity formed the research dataset for this study. The dataset contained 8,197 rows of actions performed by the students. Among these actions, those that were irrelevant according to the TF were eliminated, such as logins, updating profile, changing the role to reviewer, visits to the homepage, resulting in 5,624 data points. Then, the remaining data points were associated with the review phases and the regulation type based on the TF. The coding process adopted in this study followed a protocol similar to those recently proposed in the SRL literature [13]. According to this coding process, a macro-level construct (e.g., planning) comprises micro-level actions (e.g., setting goals or making personal plans) based on the theoretical models of SRL. In this work we follow a similar approach, where the macro-level constructs were the peer review phases and types of engagements, whereas the micro-level constructs were the actions taken by the students in Synergy. Table 2 lists all the unique actions that were considered relevant according to the TF, along with the associated phase and the regulation type.

Before the analysis, sessions were extracted from the data. A session comprises consecutive actions (of the same user) that are spaced less than 15 minutes of interval. Similar approaches have been noted in the literature where the interval of 30 minutes was used to identify the sessions [6]. The 15-minute interval was chosen 


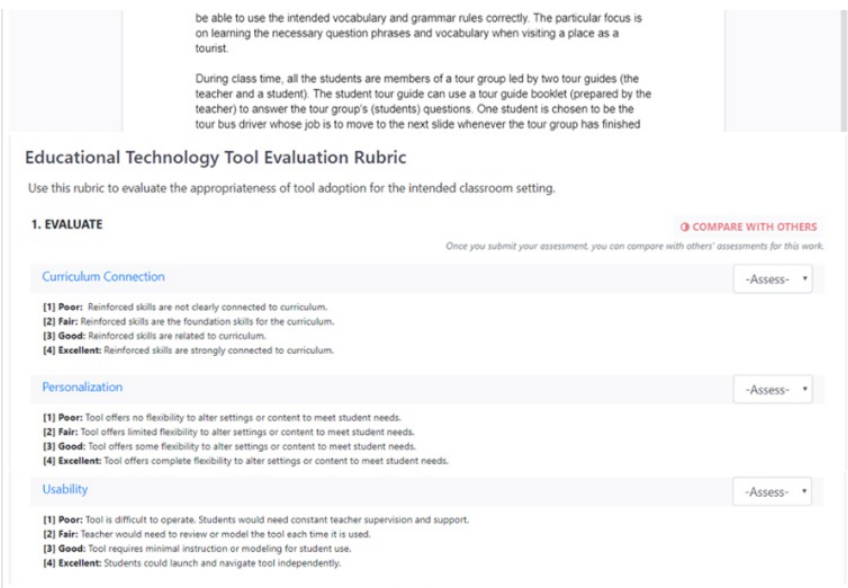

(a)

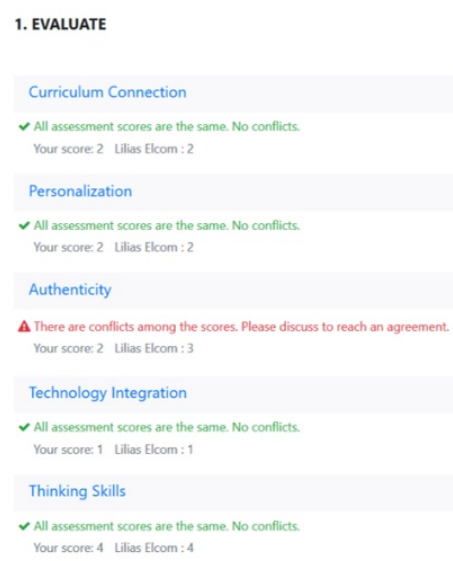

(b)

Figure 2: (a) Self-assessment interface, (b) comparison of assessment scores for the work being assessed

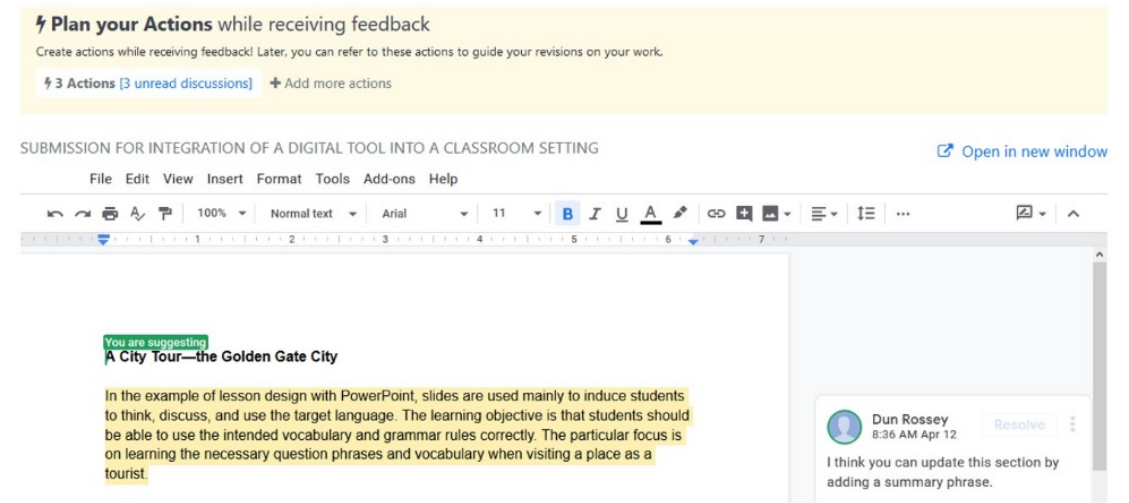

Figure 3: Feedback provision and discussion page for reviewed students

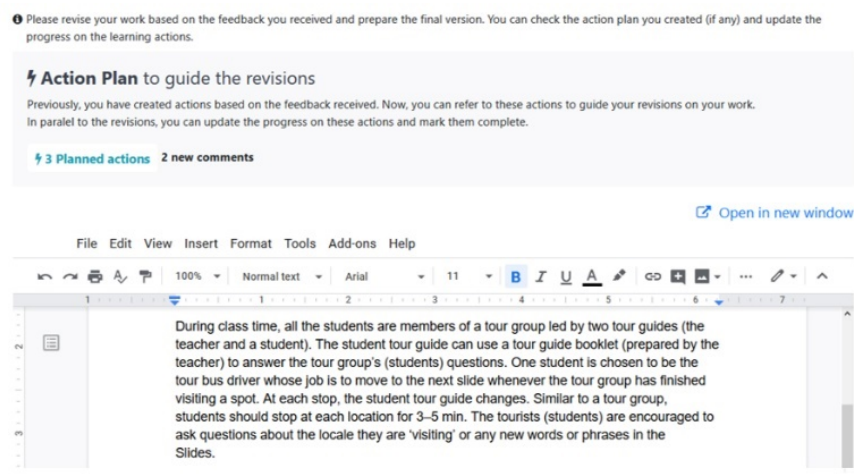

(a)

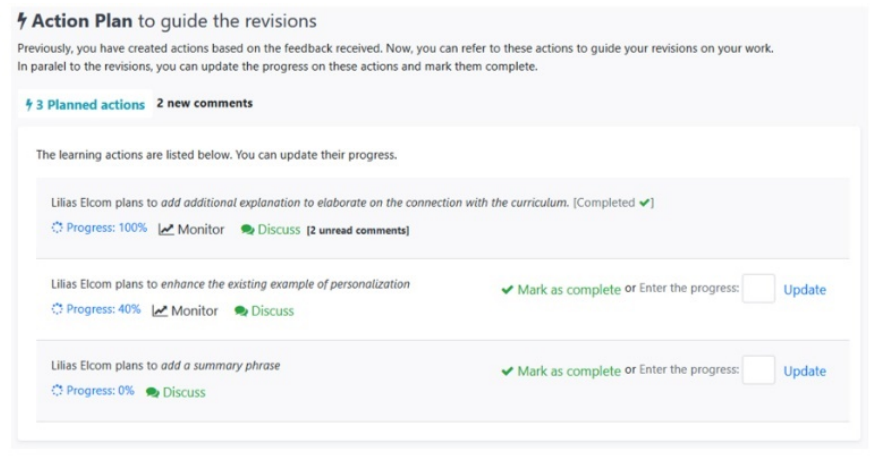

(b)

Figure 4: (a) The page for revising the work, and (b) the list of actions in the revisions page

in this study since in Synergy users are automatically logged out after 15 minutes of inactivity.

\subsection{Data Analysis}

This study aimed to discover the processes that (reviewed) students follow in a peer feedback activity. For this purpose, process mining (PM) was applied. PM algorithms are applied to log data to identify 


\section{Monitoring the Action «Go back}

\section{Changing the code about splitting strings}



Figure 5: The line chart to visualize the action progress and revision history

Table 2: Mapping between the actions, phases, and type of regulation

\begin{tabular}{|c|c|c|}
\hline Phase & Action & Regulation type \\
\hline \multirow{2}{*}{\multicolumn{2}{|c|}{$\begin{array}{l}\text { [\#1 ALIGN] Aligning the [1.1 VISIT_SELF_ASSESS] Visit self-assessment page } \\
\text { perspectives and planning[1.2 MAKE SELF ASSESS] Make a self-assessment }\end{array}$}} & SSRL \\
\hline & & SSRL \\
\hline \multirow[t]{3}{*}{ the feedback } & [1.3 COMPARE_SCORES] Compare the assessment scores for own work & SSRL \\
\hline & [1.4 VIEW_DISCUSSION] View assessment discussion for own work & SSRL \\
\hline & [1.5 POST COMMENT] Post a comment to discuss assessment scores for own work & SSRL \\
\hline \multirow{6}{*}{$\begin{array}{l}\text { [\#2 DISCUSS] Discussion } \\
\text { around the feedback to } \\
\text { support its uptake }\end{array}$} & [2.1 VISIT_FEEDBACK] Visit the feedback page & SRL \\
\hline & [2.2 POST_REPLY] Post a reply to a feedback comment & Co-RL \\
\hline & [2.3 CREATE_ACTION] Create a learning action & SRL \\
\hline & [2.4 VIEW_OWN_ACTIONS] View own learning actions created based on feedback & SRL \\
\hline & [2.5 POST_COMMENT] Post a comment to discuss a learning action owned & Co-RL \\
\hline & [2.6 VIEW_DISCUSSION] View the discussion for a learning action owned & Co-RL \\
\hline \multirow{6}{*}{$\begin{array}{l}\text { [\#3 ACT] Translation of } \\
\text { the feedback into task } \\
\text { engagement \& progress }\end{array}$} & [3.1 VISIT_REVISION] Visit the revision page & SRL \\
\hline & [3.2 VIEW_ACTIONS] View the planned learning actions along with the progress & SRL \\
\hline & [3.3 UPDATE_PROGRESS] Update the progress of a learning action & SRL \\
\hline & [3.4 POST_COMMENT] Post a comment to discuss a learning action & Co-RL \\
\hline & [3.5 VIEW_DISCUSSION] View the discussion for a learning action & Co-RL \\
\hline & [3.6 MONITOR_PROGRESS] Monitor the progress history of a learning action & SRL \\
\hline
\end{tabular}

patterns of user activity in the time axis. The use of this technique has become increasingly common in the field of SRL to determine strategies and tactics for learning [12]. In this study, process mining was applied at three different levels: phases of peer review, types of learning regulation, and micro-level actions. Besides SRL, the regulation types included SSRL and Co-RL which have been underexplored using PM in the literature.

The Disco algorithm was used to perform process mining using its implementation in the Disco ${ }^{1}$ commercial tool. The algorithm

\footnotetext{
${ }^{1}$ https://fluxicon.com/disco/
} 
was designed to handle complex processes. The tool integrates a set of metrics and filtering options to adapt the analysis to specific questions and visualizes the results in the form of a process map. In this study, two filtering options were applied: the activity threshold and the path threshold. The activity threshold determines the steps that will appear in the process based on the frequency of their occurrence. The path threshold determines the links between the student actions that should appear in the process map based on the frequency of their occurrence. While the threshold of 100 shows all the processes, the threshold of 0 brings the most frequent process. For the analysis in this study, a threshold of 100 was used to display the engagement in every activity, and a path threshold of 30 was used to keep the more frequent links, thus obtaining more interpretable process models with sufficient information.

\section{RESULTS}

The analysis follows a top-down approach, which initially explores the processes at a global level (i.e., phases of the peer review), and then moves to more detailed analyses of the types of regulation of learning, and of the actions performed within Synergy. At each level of analysis, process models were visualized for both performance groups. In these visualizations, boxes represent one of the followings depending on the level of the analysis: a peer-review phase, a regulation type or an action. The strength of the engagement with these constructs is indicated by the number inside the corresponding box and the darkness of the box color. The arrows between the boxes represent students' transition from one construct to another. The thickness of the arrows indicates the frequency of the transitions.

In Disco tool, by default the numbers inside the boxes and on the arrows indicate the absolute frequencies. Given the unequal distribution of the students across the performance levels, the relative value of each transition frequency (i.e., solid arrows) and of each box were also provided. The relative value of a transition count was calculated by dividing its count by the sum of all transition values in the same model. Similarly, the relative value of engagement count in the boxes was calculated by dividing the count by the sum of all engagement counts in the same process model. Relative values are then converted to percentages (\%), which are displayed next to their absolute values. For example, in Figure 6, it can be observed that the high performing students engaged in PHASE \#1 ALIGN 670 times. The relative value, $34.69 \%$, was calculated by: $670 /(670+748+530)$ *100.

\subsection{Process models of student engagement in peer-review phases}

The process models related to the student engagement at the level of peer-review phases are presented in Figure 6, separately for the (a) high and (b) medium performers. These models were generated based on the existing mapping of students' actions with the peer-review phases as outlined in the TF. According to the figure, the transitions between the phases were the same for both student groups. The TF suggests strong interactions between phases \#1 ALIGN and \#2 DISCUSS as well as between phases \#2 DISCUSS and \#3 ACT. These interactions were reflected in the actual student behavior as demonstrated by the mutual strong ties among them in the process model (see Figure 6). Nevertheless, the strength of the connections between the phases (based on the values attached to the lines) were somewhat different across performance groups. For the high performers, there was a slightly higher number of (reciprocal) transitions between phases \#2 DISCUSS and \#3 ACT (\#2 $\rightarrow \# 3$ : $\% 28.03$ and \#3 $\rightarrow \# 2$ : \%20.83) than between phases \#1 ALIGN and \#2 DISCUSS (\#1 $\rightarrow \# 2: \% 25.38$ and \#2 $\rightarrow \# 1: \% 20.08$ ). This difference was larger (and in the reverse direction) for the medium performers (i.e., $\# 1 \rightarrow \# 2: \% 33.75$ and $\# 2 \rightarrow \# 1: \% 24.29$ vs \#2 $\rightarrow \# 3: \% 17.86$ and $\# 3 \rightarrow \# 2$ : $\% 12.14)$. This finding suggests that the high performers put more effort in translating the feedback received into concrete actions. This behavior complies with the TF, which underlines the importance of discussing feedback to derive relevant actions and to implement them.

Despite the structural similarity, the level of engagement in each phase (indicated by the numbers inside the boxes) were different across the high and medium performers. A clear distinction between the groups is that the high performers showed a more balanced engagement in all three phases (\%34.39 in phase \#1, \%38.40 in phase $\# 2$, and \%27.21 in phase \#3). Nonetheless, the engagement level in phase \#3 ACT was relatively low for the high performers compared to their engagement in other phases, which was most probably due to the fact that the final revisions were made outside Synergy as determined by the course instructor. Therefore, the engagement in phase 3 mainly involved tracking, monitoring, and discussing the progress of the actions. On the other hand, the medium performers had a very low level of engagement in phase \#3 ACT (\%12.31) and relatively low engagement in phase \#1 ALIGN (\%34.89) compared to that in phase \#2 DISCUSS (\%52.80). This result suggests that high performers intended to participate in all phases whereas medium performers mostly paid attention to the phase \#2 DISCUSS (where they received the feedback from their peers) and mostly discarded the phase \#3 ACT (which mainly involved tracking the ongoing progress on the learning actions). Thus, the high performers behaved in alignment with the TF by balancing their participation across all phases.

Although process models built at phase level provide an understanding of overall engagement in different peer-review phases suggested by the TF, they are limited in explaining how students regulated their learning during these phases and which actions they performed in each phase (i.e., micro-level actions). The analysis results at regulation and action level are presented in the following section.

\subsection{Process models of student engagement in regulation of learning}

The process models related to the student engagement in different regulation types (i.e., SSRL, Co-RL, and SRL) are presented in Figure 7 separately for (a) high and (b) medium performers. These models were generated based on the existing mapping of students' actions with the corresponding regulation type according to the TF (see Table 2). As some of the regulation types might occur in multiple phases, one-on-one matching between the phases and the regulation of learning was not possible. Although, in the analysis, engagement in all regulation-related actions were considered, performing a single action (e.g., comparing assessment scores) does 


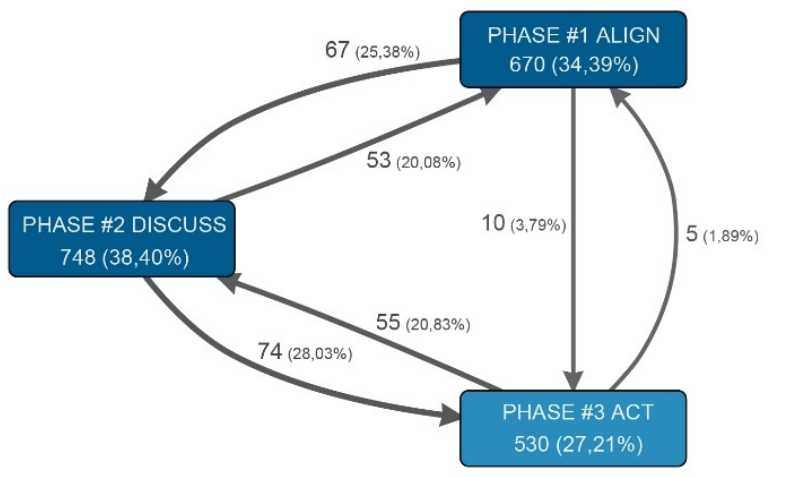

(a)

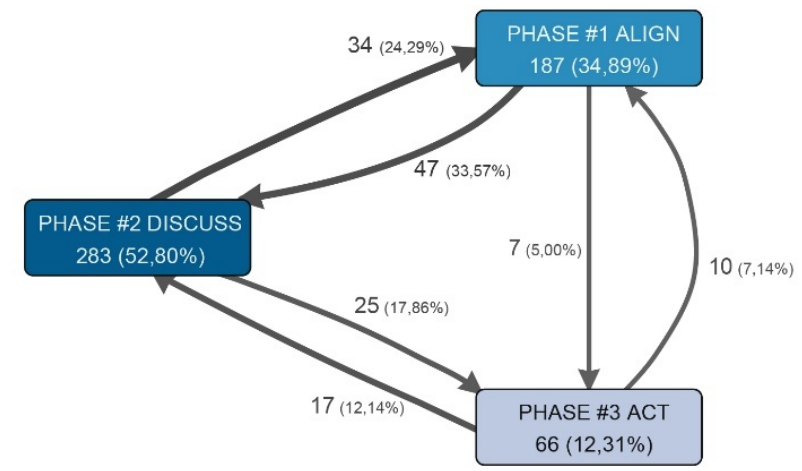

(b)

Figure 6: Process model of engagement in the phases of peer review by (a) high performers and (b) medium performers

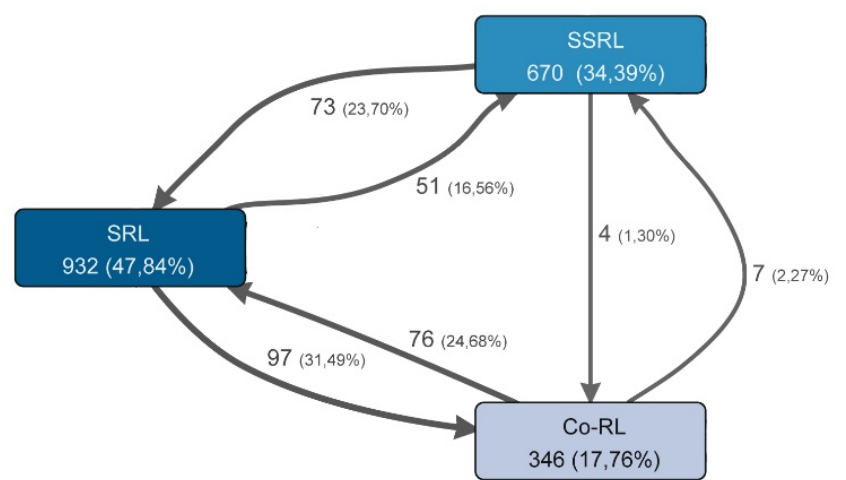

(a)

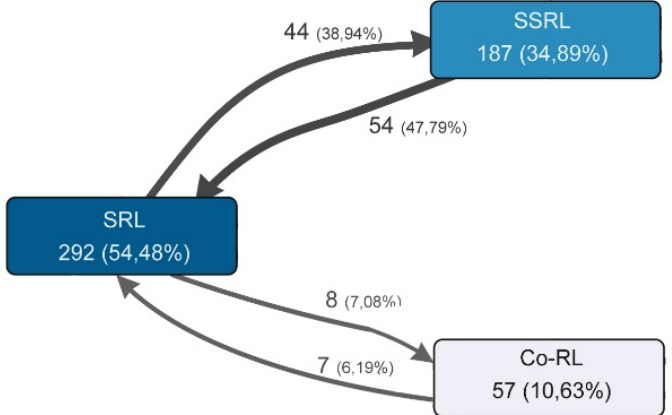

(b)

Figure 7: Process model of engagement in the regulation of learning by (a) high performers and (b) medium performers

not necessarily entail the occurrence of the regulation (e.g., SSRL). A sequence of actions (e.g., re-assessing the own work after comparing the scores) might be necessary for the regulation to take place. These action-level details are further discussed in the next section.

As it was the case for the phases, transitions among the processes were considerably similar for both student groups. The close ties between SRL and Co-RL and between SSRL and SRL were expected according to the TF. However, the strengths of the connections were distinctive across performance groups. For the high performers, there was a reciprocal connection between SRL and Co-RL (SRL $\rightarrow$ Co-RL: \%31.49, Co-RL $\rightarrow$ SRL: \%24.68), which was quite weak for the medium performers (SRL $\rightarrow$ Co-RL: \%7.08, Co-RL $\rightarrow$ SRL: \%6.19). As suggested by the TF, this connection (existing for the high performers) indicates a positive influence of peer support on helping students regulate their learning. Similarly, a strong interaction was observed between SRL and SSRL in both student groups (for the high performers, SSRL $\rightarrow$ SRL: \%23.70, SRL $\rightarrow$ SSRL: \%16.56; for the medium performers, SSRL $\rightarrow$ SRL: \%47.79, SRL $\rightarrow$ SSRL: \%38.94), aligned with the TF. This interaction suggests the positive impact of students' participation in aligning perspectives about the quality of the work (i.e., SSRL) on their internalization of the feedback received and the subsequent efforts to make use of it such as generating actions (i.e., SRL). Interestingly, for the high performing students, the transitions from SRL to Co-RL were relatively more often than those from SRL to SSRL, which indicates the cyclic dynamic relation between Co-RL and SRL.

Moreover, relative engagement levels in the regulation types were very similar across performance levels. Students highly engaged in SRL (high performers: \%47.84, medium performers: \%54.48) followed by SSRL (high performers: \%34.39, medium performers: \%34.89), whereas the engagement in Co-RL was relatively low (high performers: \%17.76, medium performers: \%10.63). This result indicates that students regulated their learning based on the feedback, but they did not interact much with the reviewing peers to discuss the feedback and ask for help about the actions they derived from the feedback. Nonetheless, low level of Co-RL engagement did not seem to have an effect on the final project grade. This finding conflicts with the TF which recommends the importance of Co-RL engagement. The following section provides further insights into engagement in learning regulation based on micro-level student actions. 




Figure 8: Process model of the engagement in the activities by high performers

\subsection{Process models of student engagement in micro-level actions}

The process models for each performance group are presented in Figure 8 and Figure 9. In these models, normalization was applied separately for the numbers in the boxes of 1.1 VISIT_SELF_ASSESS, 2.1 VISIT_FEEDBACK, and 1.3 COMPARE_SCORES (which are the main pages in Synergy representing each phase) and for the actions that can be performed under each page. This separate normalization process aimed at helping compare the relative engagement levels among the main pages and identify the prominent (or unimportant) actions within each page. According to the models, the processes engaged and the connections among them are found to be substantially different between the performance groups, which is elaborated in the following two sections.

4.3.1 High Performers. According to the process model shown in Figure 8, for high performers, among three main pages, visiting the feedback page (the main page for the 2nd phase in the TF) was the most prominent action (\%49.37) and it played an intermediary role between visiting the assessment page (main page for the 1st phase in the TF) and visiting the revision page (the main page for the 3rd phase in the TF). This global process structure suggests that the high performers' overall engagement in the review processes within Synergy complied with the underlying TF.

Considering the engagement in the actions on the assessment page, a very close alignment is noted between the path followed by high-performing students and the structure suggested by the TF. The students engaged in every action available on the assessment page, with comparing assessment scores (1.3 COMPARE_SCORES) being the most frequent (47.18\%). The process model shows that, after visiting the self-assessment page (1.1 VISIT_SELF_ASSESS: $24.47 \%$ ), students either assessed their work (1.2 MAKE_SELF_ASSESS: $22.58 \%$ ) or (if the self-assessment is already done) they compared their self-assessment scores with those assigned by others (1.3 COMPARE_SCORES: $47.18 \%$ ). This mutual connection between 1.2 and 1.3 suggests that students might have reflected on the accuracy of their self-assessments after checking the scores assigned by others and decided to update the assessment scores. This activity pattern provides evidence regarding the engagement of the high performers in SSRL as hypothesized in the TF. This finding is further supported by the students' subsequent actions. Afterwards, they viewed the discussions on the assessment scores (1.4 VIEW_DISCUSS: $25.60 \%$ ) and made a discussion post (1.5 POST_COMMENT: $4.64 \%$ ), from which they transitioned to comparing scores and eventually self-assessing their work. This transition suggests that the students engaged with the discussions around the scores they received and then decided to modify their self-assessment, and thus socially regulating their learning.

The high performers' engagement in the actions on the feedback page mostly followed the theorized process structure. After visiting the feedback page (2.1 VISIT_FEEDBACK: 49.37\%), where the work 
being assessed was displayed as an embedded Google Doc with feedback provided as comments, the students engaged in creating learning actions based on peer feedback received (2.3 CREATE_ACTION: $13.85 \%)$, listing all actions created (2.4 VIEW_OWN_ACTIONS: $34.51 \%)$, and checking the discussions on these actions if any $(2.6$ VIEW_DISCUSSION: $12.09 \%$ ). These transitions suggest that after checking the feedback provided, the students cared about creating an action plan to organize the revisions that they planned to implement, which is an SRL activity hypothesized to take place by the TF. Associated with this, the students were also highly engaged in reflecting on/discussing the feedback they received by posting replies to the feedback comments on the Google Doc (2.2 POST_REPLY: $39.04 \%$ ). That is, the students were active in making sense of peer feedback so that they could derive relevant learning actions, which is considered a critical element of peer review by the TF. As the students were able to open the Google Doc outside Synergy to post replies any time, the connection of this process (post a reply to feedback) with visiting the feedback page could not be reflected properly in the process model. Students rarely posted comments to discuss their actions (2.5 POST_COMMENT: 0.50\%) although they tended to view the discussion comments on the actions $(2.6$ VIEW_DISCUSSION: $12.09 \%$ ). This result further supports the previous finding (section 4.2) regarding the low level engagement in Co-RL.

Moreover, the high performers' engagement patterns on the revision page align with the TF. The most predominant action after visiting the revision page (3.1 VISIT_REVISION: $26.16 \%$ ) was to view the planned actions (3.2 VIEW_ACTIONS: 42.73\%) (where Synergy also displays the current progress on each action listed). Afterwards, the students updated the action progress (3.3 UPDATE_PROGRESS: $12.50 \%$ ). This suggests that the high performing students were inclined to check the progress on the actions and to update their progress to properly regulate their learning as theorized by the TF. The students also checked the ongoing discussions (3.5 VIEW_DISCUSSION: $33.72 \%$ ) and posted some comments to discuss their ongoing progress on the actions (3.4 POST_COMMENT: $7.27 \%$ ). This may indicate the students' intentions to benefit from the discussions with the peers to enhance some of the changes they incorporated or to further improve their work. In other words, some level of engagement in Co-RL was noted by high performers. Engagement in monitoring the progress history on the actions (3.6 MONITOR_PROGRESS: 3.78\%) was quite low. For monitoring, Synergy provides a line chart to demonstrate the progress on the selected action over time. The low level of monitoring could be associated with the difficulty reading the visualization and making sense of it.

4.3.2 Medium performers. In contrast to the close alignment between the behavior of the high performers and the TF, the medium performers' engagement was loosely connected with what was theorized. According to the process model shown in Figure 9, visiting the feedback page (2.1 VISIT_FEEDBACK: 53.11\%) was also the central process. The structure of the model is highly disorganized in contrast to that of high performers where the intermediary role of phase \#2 DISCUSS between phase \#1 ALIGN and phase \#3 ACT was highly notable and the actions within each phase were well grouped and connected.
The engagement in the first phase of the reviews was mostly limited to two actions: visiting the self-assessment page (1.1 VISIT_SELF_ASSESS: $31.37 \%$ ) and performing a self-assessment (1.2 MAKE_SELF_ASSESS: 56.98\%). Students also compared the assessment scores (1.3 COMPARE_SCORES: 37.21) after they completed the self-assessment. However, after reflecting on the assessment scores, they did not attempt to repeat the self-assessment again to resolve the discrepancies in the perspectives about the quality of the work. Additionally, they barely engaged in discussions of the assessment scores. These results indicate that no SSRL took place among the medium performers during the first phase although they engaged in several related processes such as self-assessment and comparing the assessment scores.

The revisions page was the least visited (3.1 VISIT_REVISION: $15.53 \%$ ), indicating minimal engagement in phase 3 . On this page, the only process with which the students engaged was to view the planned actions (3.2 VIEW_ACTIONS). The students did not take any other related actions on the revisions page (e.g., updating action progress, posting a discussion comment, or monitoring ongoing progress). Thus, the students barely engaged in Co-RL or SRL in the last phase. These results indicate that the low level of engagement in Co-RL and SRL may negatively affect student performance, as suggested by the TF.

\section{DISCUSSION}

The results of this study provide evidence about the benefits of mutually connecting theory and LA. The theory helped (a) identify the relevant data points (which were specific actions taken by students in Synergy) from the large clickstream data, (b) match these data points with the phases of the peer review, and (3) determine their associations with the particular type of learning regulation. In other words, theory helped identify where to focus within the clickstream data and provided a context for understanding how the chosen data points are related with students' peer-review engagement. Moreover, grounding learning analytics in a theory enabled us to follow a layered approach in the analysis. While the top layer (i.e., phases of the peer review) presented a global perspective on student engagement across different performance levels, the intermediate layer (i.e., regulation types) provided a deeper understanding of how the students regulated their learning, and the bottom layer (i.e., actions) helped identify the patterns of engagement in micro-level actions. The findings obtained at each level of the analysis contributed to the existing understanding of peer-review engagement from different perspectives.

Theory also played a critical role in identifying a priori engagement patterns that were expected to happen. In this way, theory helped obtain an informed perspective when interpreting the results of process mining. The resulting process models mostly differed based on students' performance levels. Consistently, the high performers' engagement patterns were closely aligned with the TF whereas the medium performers' engagement largely deviated from what was theorized. In particular, the high performing students tended to be more active in reflecting on their self-assessment and aligning their perspectives with the reviewing peers during the first phase (\#1 ALIGN), where the medium performers' engagement in SSRL was minimal. Moreover, the high performers were more 


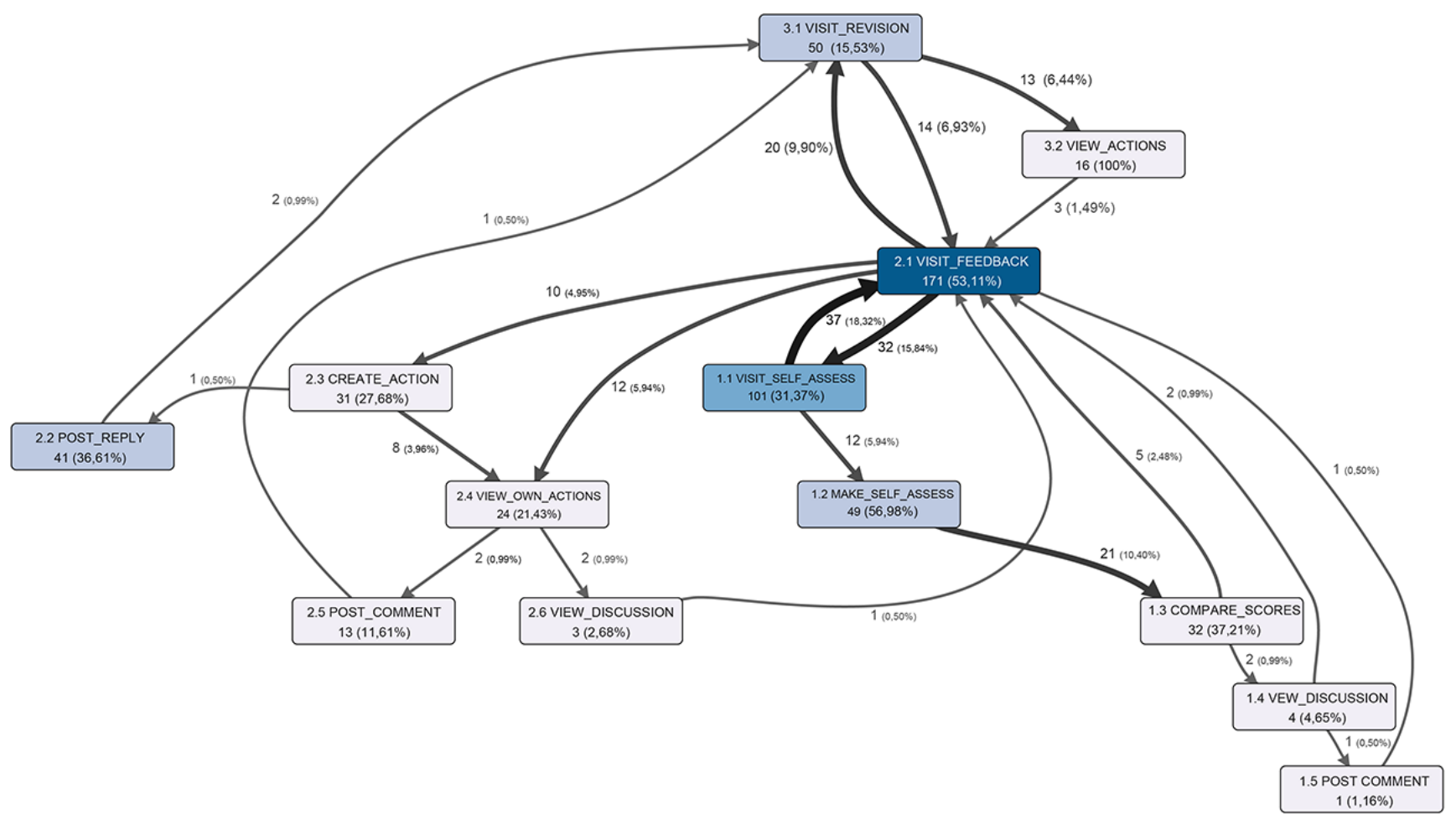

Figure 9: Process model of the engagement in the activities by medium performers

engaged with checking and tracking their progress on the learning actions (i.e., SRL) compared to the low performers. Similar findings were also noted in the literature [12], suggesting that optimal learners plan and construct more cohesive patterns of learning aligned with the grounding theory. Although the grounding theory was mostly supported by the engagement patterns of the high performers, several unexpected findings were also noted. The low level of Co-RL engagement did not seem to have an effect on student performance, which was against the previous work reporting the effectiveness of Co-RL [14]. For example, the results in [15] showed that students who received the web-based Co-RL attained significantly better computing skills than those who received the traditional teaching method. Additionally, monitoring, which is an essential element of SRL, was rarely performed by the students. These findings derived from the process models may suggest some reconsideration of the model and its operationalization in Synergy. Thus, learning analytics driven by theory informs back the theory.

The approach used in this study helped detect engagement processes not only in SRL but also in SSRL and Co-RL, which are understudied constructs in the learning analytics literature. Similar to previous findings [12], high performance was closely associated with engagement in all regulation types. Accordingly, the engagement patterns pertaining to the high performing students were much aligned with the theorized sequences of the micro-level actions, whereas the medium performing students seldom engaged in the processes regarding the regulation of learning. Moreover, the findings of this study underline the existence and the importance of the cyclic dynamic relations between different types of regulation [7]. In the high performing students' engagement patterns, a high number of bidirectional transitions occurred between SRL and SSRL and between SRL and Co-RL. These frequent transitions suggest that different regulation types may feed each other and lead to a higher performance. Therefore, embedding specific support mechanisms into learning activities to promote engagement in SRL, SSRL, and Co-RL can lead to higher learning gains and achievement.

There are several implications of this study. First, the learning design enacted within Synergy and by the instructors when implementing a peer review activity can provide a less flexible structure that enforces a certain path of learning, as suggested by the grounding theory. Associated with this point, Synergy could derive indicators that students are deviating from "successful" paths and advice students about this situation (or warn the instructors). In other words, learning analytics tools can be integrated into Synergy to guide student engagement during peer reviews. Moreover, Synergy, and its grounding theory, provide design guidelines on how to effectively support peer review situations that might also be supported by learning environments different from Synergy. But, more importantly, the results from this research work also suggest that certain learning activities, within peer review learning designs, are key for generating the data needed to obtain learning analytics indicators about significant regulatory processes. This implication reinforces the importance of aligning learning design and learning analytics [4]. An important implication for research is that the findings of this research suggest theory-based indicators to 
measure engagement not only in SRL but also in SSRL and Co-RL. These indicators can be used to build predictive models on student engagement in peer reviews (and other collaborative activities).

\section{CONCLUSION}

In this paper, we present a theory-based learning analytics approach to identifying engagement patterns in a peer review activity facilitated via a web-based tool called Synergy. This study makes a strong case for grounding learning analytics in theory [11]. Some limitations of this study should be acknowledged. First, the results presented in this study come from a single course with low enrollment, which limits the generalizability of the findings to other contexts. To support the current findings with further evidence, we plan to conduct future studies in larger and different contexts. The second limitation is that only a single type of research data was used, which although helped identify engagement patterns, was limited in explaining why students (did not) behaved in certain ways leading to the observed patterns. Qualitative methods could provide supplementary data about students' experiences, perceptions, and intentions. Future work should administer such methods as interviews or open-ended surveys to investigate the reasons behind the engagement patterns. Furthermore, in the present study, only the reviewed students' activities were analyzed, and the possible effects of students' (lack of) interactions with group members and reviewing peers were not examined. However, given the dialogiccollaborative nature of the proposed feedback process, a joint future study of both reviewed and reviewing students in addition to the interactions with teammates might provide a better understanding of the global process and the interactions between students.

\section{ACKNOWLEDGMENTS}

The research of the first author has been fully funded by the European Union's Horizon 2020 research and innovation programme under the Marie Sklodowska-Curie grant agreement 793317. The research of the other authors is partially funded by the European Regional Development Fund and the National Research Agency of the Spanish Ministry of Science, Innovations and Universities under project grant TIN2017-85179-C3-2-R, by the European Regional Development Fund and the Regional Council of Education of Castile and Leon under project grant VA257P18, and by the European Commission under project grant 588438-EPP-1-2017-1-EL-EPPKA2-KA.

\section{REFERENCES}

[1] RolaAjjawi and David Boud. 2017. Researching feedback dialogue: An interactional analysis approach. Assess. Eval. High. Educ.42, 2 (2017), 252-265. DOI:https://doi.org/10.1080/02602938.2015.1102863

[2] M.Bannert, P. Reimann, and C. Sonnenberg. 2014. Process mining techniques for analysing patterns and strategies in students' self-regulated learning. Metacognition Learn.9, 2 (2014), 161-185. DOI:https://doi.org//https://doi.org/10.1007/ s11409-013-9107-6\}

[3] ErkanEr, Yannis Dimitriadis, and Dragan Gašević. 2020. A collaborative learning approach to dialogic peer feedback: a theoretical framework. Assess. Eval. High. Educ.Online, (2020).

[4] ErkanEr, Eduardo Gómez-Sánchez, Yannis Dimitriadis, Miguel L. Bote-Lorenzo, Juan I. Asensio-Pérez, and Susana Álvarez-Álvarez. 2019. Aligning learning design and learning analytics through instructor involvement: A MOOC case study. Interact. Learn. Environ.27, 5-6 (2019), 685-698. DOI:https://doi.org/10.1080/ 10494820.2019.1610455

[5] C.W. Günther and W. M. P. van der Aalst. 2007. Fuzzy mining - Adaptive process simplification based on multi-perspective metrics. In Business Process Management, 328-343. DOI:https://doi.org/10.1007/978-3-540-75183-0_24.

[6] JelenaJovanovi, Dragan Gasesvic, Shane Dawson, Abelardo Pardo, and Negin Mirriahi. 2017. Learning analytics to unveil learning strategies in a flipped classroom. Internet High. Educ.33, (2017), 74-85. DOI:https://doi.org/10.1016/j.iheduc. 2017.02.001

[7] JonnaMalmberg, Sanna Järvelä, and Hanna Järvenoja. 2017. Capturing temporal and sequential patterns of self-, co-, and socially shared regulation in the context of collaborative learning. Contemp. Educ. Psychol.49, (2017), 160-174. DOI:https: //doi.org/10.1016/j.cedpsych.2017.01.009

[8] MulderR. A., Pearce J. M., and Baik C. 2014. Peer review in higher education: Student perceptions before and after participation. Act. Learn. High. Educ.15, 2 (2014), 157-171. DOI:https://doi.org/10.1177/1469787414527391

[9] RaoulMulder, Chi Baik, Ryan Naylor, and Jon Pearce. 2014. How does student peer review influence perceptions, engagement and academic outcomes? A case study. Assess. Eval. High. Educ.39, 6 (2014), 657-677.

[10] DavidNicol, Avril Thomson, and Caroline Breslin. 2014. Rethinking feedback practices in higher education: a peer review perspective. Assess. Eval. High. Educ.39, 1 (2014), 102-122. DOI:https://doi.org/10.1080/02602938.2013.795518

[11] PeterReimann. 2016. Connecting learning analytics with learning research: The role of design-based research. Learn. Res. Pract.2, 2 (2016), 130-142. DOI:https: //doi.org/10.1080/23735082.2016.1210198

[12] J.Saint, Whitelock-Wainwright A., D. Gasevic, and A Pardo. Trace-SRL: A framework for analysis of micro-level processes of self-regulated learning from trace data. IEEE Trans. Learn. Technol.DOI:https://doi.org/\{https://doi.org/10.1109/ TLT.2020.3027496\}

[13] M.Siadaty, D. Gasevic, and M. Hatala. 2016. Trace-based micro-analytic measurement of self-regulated learning processes. J. Learn. Anal.3, 1 (2016), 183-214.

[14] Chia-wenTsai. 2015. The effect of online co-regulated learning in the implementation of team-based learning on improving students' involvement. High. Educ. Res. Dev.34, 6 (2015), 1270-1280. DOI:https://doi.org/10.1080/07294360.2015.1024631

[15] Chia-wenTsai. 2015. Applying web-based co-regulated learning to develop students' learning and involvement in a blended computing course. Interact. Learn. Environ.23, 3 (2015), 344-355. DOI:https://doi.org/10.1080/10494820.2013.764323

[16] N.E. Winstone, R. A. Nash, M. Parker, and J. Rowntree. 2017. Supporting learners' agentic engagement with feedback: A systematic review and a taxonomy of recipience processes. Educ. Psychol.52, 1 (2017), 17-37. DOI:https://doi.org//https: //doi.org/10.1080/00461520.2016.1207538\}

[17] AlyssaFriend Wise and David Williamson Shaffer. 2015. Why theory matters more than ever in the age of big data. J. Learn. Anal.2, 2 (2015), 5-13. 